\title{
Effects of training level on reversal and extradimensional shifts
}

GEORGE R. POTTS AND B. R. HERGENHAHN

HAMLINE UNIVERSITY
Reversal (RS) and extradimensional (EDS) shift groups were subdivided into undertrained (UT), criterion trained (CT), and overtrained (OT) conditions. Results indicated: (1) The RS was leamed faster than the EDS in both the CT and OT conditions, but Ss in the IIT conditions did not differ. (2) Ss in the IIT condition took longer to learn the RS than Ss in either the CT or OT conditions, but no significant difference was found between the CT vs OT conditions. (3) The EDS group did not differ significantly with training level. The ORE was demonstrated, but the hypothesis that this ORE might be explained in terms of the increased awareness of incidental cues which has been found in OT was not supported.

Considerable experimental investigation has been evoked by the finding that overtraining on a discrimination problem tends to facilitate the learning of its reversal. The phenomenon has been referred to as the Overtraining Reversal Effect (ORE). Many attempted explanations of the ORE have revolved around the hypothesis that during training $S$ learns to respond to the relevant stimulus dimension and that during overtraining his tendencies of responding to only that dimension become fixated, thus assuring continued attention to that dimension in the reversal (RS) situation [Sutherland (as reported in Mackintosh, 1963); Mackintosh, 1962; Spence (in personal communication to Reid, 1953)].

Recent findings (Bahrick, 1957; Hergenhahn \& Lee, 1965), however, have shown evidence that most incidental learning seems to occur in the beginning stages of learning and in the latter stages of overlearning. This would seem to indicate increased attention to incidental cues during over- and underlearning, and would thus be in direct disagreement with the hypothesis that overtraining tends to focus S's attention on the relevant dimension at the expense of awareness of other cues. Another possible explanation (Hergenhahn, 1966) is that the decrease in the probability of responding to irrelevant stimuli for overtrained Ss is due not to a decrease in the awareness of incidental cues, but rather to an increased awareness of all cues, relevant and incidental, and a resulting increased awareness of their interrelations.

If, indeed, the ORE can be explained by an increased attention to incidental cues that comes with overtraining, and if a similar increase exists in early trials, it should be possible to illustrate the existence of an Undertraining Reversal Effect (URE). The present experiment utilizes a conditional discrimination problem to examine the effects of training level on both a reversal (RS) and an extradimensional (EDS) shift.

\section{Method}

Eighty $8 \times 5$ in. cards were employed. Each card bore a circle and a triangle, one on the right side and one on the left. One of these figures was outlined in red, the other in black. They were imposed upon a background of parallel lines which were either oblique or vertical and either blue or red. All 16 possible combinations of these four conditions (color and position of the figures and direction and color of the lines) were equally represented. The cards were randomized by shuffling at frequent intervals.

Sixty Hamline undergraduates were divided into two groups according to whether they were to receive a RS or an EDS. Each of these groups was subdivided into three groups of $10 \mathrm{Ss}$ according to training level. An additional $15 \mathrm{Ss}$ were eliminated for failure to reach criterion within an arbitrarily chosen limit of 70 trials.

Each $S$ was seated in front of a table, shown the series of cards one at a time, and instructed to point to whichever of the figures he felt was correct. If he made the proper choice, E said "correct;" if he did not make the proper choice, E said "wrong."

There were three types of problems: (1) When the lines were blue, the black figure was correct; when the lines were red, the red figure was correct. (2) When the lines were blue, the red flgure was correct; when the lines were red, the black figure was correct. (3) When the lines were oblique, the triangle was correct; when the lines were vertical, the circle was correct (EDS).

The Ss in each of the RS conditions were counterbalanced, half being reversed from (1)-(2) and the other half from (2)-(1). Similarly, half the Ss in the EDS conditions were shifted from (1)-(3) while half were shifted from (3)-(1).

The undertrained (UT) Ss were shifted after four, the criterion trained (CT) Ss after eight, and the overtrained (OT) Ss after 32 consecutive correct choices. Criterion for learning of the shift was defined as eight consecutive correct choices.

\section{Results}

Mean trials to criterion for the UT, CT, and OT conditions of the RS were $25.0,9.6$, and 1.9 , respectively; for the EDS, these values were $25.3,26.8$, and 27.3 , respectively.

The EDS was found to require significantly more trials to criterion than the $R S(F=10.73$, df $=1 / 54$, $p<.001)$. The interaction just missed being significant at the .05 level $(F=2.86, \mathrm{df}=2 / 54, .05<\mathrm{p}<.10)$. Using the Duncan multiple range test, significant 


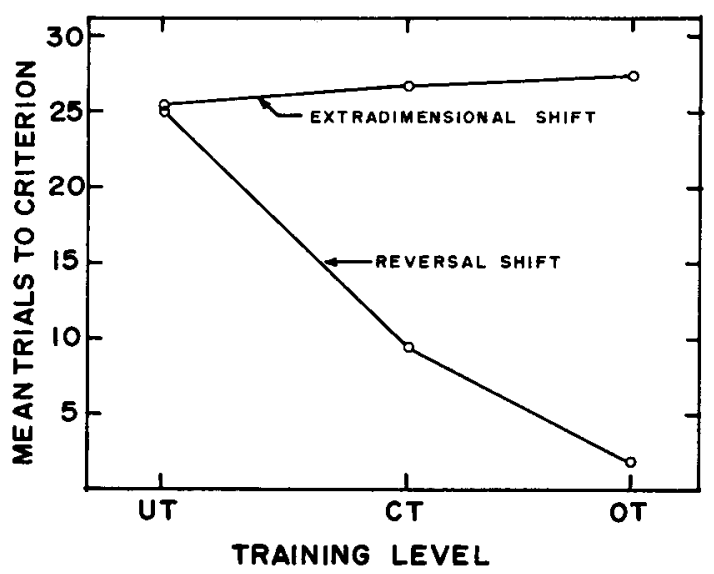

Fig. 1. Effects of training level on the speed of leaming reversal and an extradimensional shift.

differences were found between the RS vs EDS groups in both the CT and OT conditions $(p<.05, p<.005)$, but the mean trials to criterion in the UT conditions were almost identical. Ss in the UT conditions took significantly longer to learn the RS than either CT or OT Ss $(p<.05, p<.01)$, but the difference in the CT vs OT Ss was not significant. Mean trials to criterion for all groups during the shift condition are shown in Fig. 1.

A Mann-Whitney $U$ test found that the UT groups required significantly more trials to learn the shift than the other groups took to learn the original problem $(z=2.30, p=.01)$. The OT groups learned the $R S$ significantly faster than the original problem $(z=3.45$, $\mathrm{p}<.001)$.

\section{Discussion}

It is very difficult to define UT, CT, and OT. The extreme variability of the CT group is to be expected, for at that level some Ss will be UT, some CT, and some OT. Indeed, the sharp reduction in variability for OT Ss seems to be as sensitive a measure of the ORE as the number of trials to criterion. It should also be noted that although it is possible that some UT Ss reached criterion by chance alone with no learning involved, the fact that this group took significantly longer to learn the RS than the other groups took to learn the original problem definitely indicates that some other factor was involved.

Hergenhahn's (1966) hypothesis that the ORE might be explained by an increased awareness to incidental cues found in OT was not supported, for in spite of evidence that this awareness is also high in UT, no corresponding facilitation of the RS was found. It should be noted, however, that the hypothesis that OT tends to fixate attention on only the relevant cue was not supported either, for if such were the case, one would predict the learning of the EDS to be considerably more difficult for OT Ss. Training level, instead, was found to have no significant effect on the learning of the EDS. Bruner (1957) hypothesized that OT may serve to fix S's attention not on the relevant dimension but, rather, on the modality in which the primary discrimination had been made. This could explain why training level did not have a significant effect on the learming of the EDS, both parts of which were visual discriminations. An expansion of the present study which included an EDS involving two different modalities would serve to test this hypothesis.

\section{References}

Bahrick, H. P. Incidental learning at five stages of intentional learning. J. exp. Psychol., 1957, 54, 259-261.

Bruner, J. S. Comment on Effect of overtraining on subsequent learning of incidental cues. Psychol. Rep., 1957, 3, 317-320.

Hergenhahn, B. R. Effects of overtraining on discrimination reversal learning. Unpublished doctorate thesis, University of Arizona, 1966.

Hergenhahn, B. R., \& Lee, P. Influence of degree of intentional learning upon performance of an incidental task. Psychol. Rep., $1965,16,781-785$.

Mackintosh, N. J. The effects of overtraining on a reversal and and nonreversal shift. J. comp. physiol. Psychol., 1962, 55. 555-559.

Mackintosh, N. J. Extinction of a discrimination habit as a function of overtraining. J. comp. physiol. Psychol., 1963, 56, 842-847.

Reid, L. S. The development of noncontinuity behavior through continuity learning. J. exp. Psychol., 1953, 46, 107-112. 